\title{
An Improved Sequential Initiation Method for Multitarget Track in Clutter with Large Noise Measurement
}

\author{
Daxiong Ji and Dongdong Li \\ Shenyang Institute of Automation, Chinese Academy of Sciences, Shenyang 110016, China \\ Correspondence should be addressed to Daxiong Ji; jdx@sia.cn
}

Received 5 June 2014; Revised 24 July 2014; Accepted 30 July 2014; Published 14 August 2014

Academic Editor: Davide Spinello

Copyright (C) 2014 D. Ji and D. Li. This is an open access article distributed under the Creative Commons Attribution License, which permits unrestricted use, distribution, and reproduction in any medium, provided the original work is properly cited.

\begin{abstract}
This paper proposes an improved sequential method for underwater multiple objects tracks initiation in clutter, estimating the initial position for the trajectory. The underwater environment is complex and changeable, and the sonar data are not very ideal. When the detection distance is far, the error of measured data is also great. Besides that, the clutter has a grave effect on the tracks initiation. So it is hard to initialize a track and estimate the initial position. The new tracks initiation is that when at least six of ten points meet the requirements, then we determine that there is a new track and the initial states of the parameters are estimated by the linear least square method. Compared to the conventional tracks initiation methods, our method not only considers the kinematics information of targets, but also regards the error of the sonar sensors as an important element. Computer simulations confirm that the performance of our method is very nice.
\end{abstract}

\section{Introduction}

Multitarget tracking plays a significant role in many areas of engineering such as surveillance [1], computer vision [2,3], network and computer security [4], and robot [5]. Targets arise randomly in space and time, persist for a random length of time, and then cease to exist; the sequence of states that a target follows during its lifetime is called a track. The positions, or more generally partial states, of moving targets are measured, either at random intervals or, more typically, in periodic scans that measure the positions of all targets simultaneously. The position measurements are noisy and there is a heavy background in spurious measurements, that is, false alarms or clutter. Filtering techniques joined with data association algorithms have been developed to sequentially estimate the target tracks using uncertain origin receiver observations. However, many tracking techniques require estimation of the targets' initial states, number of existing targets, and validation of the estimated tracks, which are challenging tasks in the presence of clutter and noise. The principal initial states include the relative position or absolute position and the relative velocity or absolute velocity. Track initiation and confirmation has been an active research area and various methods are published in literature. These methods can roughly be divided into sequential methods and batch processing techniques.

The heuristic method and the logic-based method involve the processing of a sequence of measurements received during consecutive sonar scans. The sequence of measurements represents the input to a time-window containing $N$ sonar scans. When the number of detections contained in the window reaches a specified value, a successful track initiation is obtained; otherwise the window is moved one scan towards the right, that is, for increasing time. While the heuristic method uses two simple rules, namely, velocity and acceleration constraints, to reduce potential tracks for initiation, the logic-based method uses prediction and gating to identify potential tracks in a multiple hypothesis fashion [6]. Logicbased $M$-out-of- $N$ algorithm is a common and straightforward track initiation algorithm which is appropriate for environments with low false alarm rate. Performance analyses of various $M$-out-of- $N$ algorithms have been reviewed in $[7,8]$.

For the batch technique approach, measurements from the past $N$ scans are processed simultaneously to determine feasible target trajectories. The $N$ scans of data are treated as 
an image, and the tracks are initiated if some curves (usually straight lines) are detected. The HT transforms a measurement to a curve in a parameter space, and a straight line in the $x-y$ coordinate will generate a set of curves intersecting with each other at a common point in the parameter space [9]. A potential track is then determined by detecting the peak in the parameter space. For targets having curved trajectories, they will be approximated as a straight line over reasonably short time intervals. However, the standard HT which utilizes the histogram approach suffers from some shortcomings for the tracking applications [10]. A modified version of Hough transform is outlined in [11] and compared with $M$-out-of- $N$ and Hough transform algorithms in [12].

The underwater environment is complex and changeable, sonar data are not very ideal, and the measurement error is also great. After simulating with the heavy noise data, we realize that the above four methods cannot initialize the real trajectory. That is to say, the conventional tracks initiation methods are not suitable for the hostile underwater environments.

This paper takes up the challenge of sequential-based track initiation in the multiple targets tracking problem on range and bearing observation. We introduce a novel sequential methodology for track initiation. The new algorithm derives from the conventional logic-based track initiation process in order to deal with the heavy noise measurement. At last, we demonstrate that the new method can initiate the trajectory in time through simulation, in the hostile underwater environment.

The structure of this paper is as follows. Section 2 introduces the hostile underwater environment; Section 3 gives a brief description of the track initiation techniques of rulebased, logic-based, and Hough transform method, and we analyze why the conventional methods cannot deal with the heavy noise measurement. Then the improved sequential initiation method is proposed in Section 4. Computer simulations are also performed in Section 5.

\section{Underwater Measurement}

The special underwater environment, such as long time delay, great measurement noise, heavy clutter, and a lot of unknown interference, may have an important effect on the tracks initiation. So it is necessary for us to basically learn about the underwater environment. Then for the special underwater environment, we will analyze and demonstrate why the conventional methods cannot deal with the heavy noise measurements.

2.1. Long Time Delay and Less Data. In the underwater environment, the sound velocity is much smaller than the light velocity. Sound velocity is about $1500 \mathrm{~m} / \mathrm{s}$ in the water. So if the distance between a target and the sonar sensor is $6000 \mathrm{~m}$, the active sensor emits the sound wave, detects the target, and returns. The time of the whole process is approximately $8 \mathrm{~s}$. During the detecting process, the target has at least $4 \mathrm{~s}$ to take action to avoid our tracking. That is to say, if the velocity of the target is $5 \mathrm{~m} / \mathrm{s}$, then, the estimated

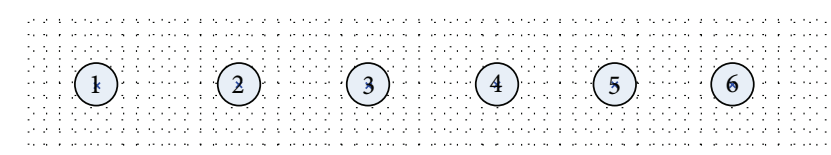

Figure 1: Distribution of ideal detection.

position will be a deviation of $20 \mathrm{~m}$ compared to the actual position. This is already in the ideal situation. In the majority of situations, the error of estimation is much bigger.

Long time delay means the available data are relatively less. In the above case, the sampling period is $12 \mathrm{~s}$. In more detail, only five data can be used for us to initialize a new trajectory in a minute. In more conditions, in order to detect much farther targets, the sampling period is a few seconds. So it is different from the video information and optical information; data in the underwater environment is very few.

2.2. Strong Noise. The performance of the sonar also affects the result of measurements. Sound travels much slower than the electromagnetic wave; so we cannot get enough accurate data with high frequency of detection. In the underwater environment, the farther the distance between the sonar and the target is, the longer the detection delay is and the worse the measurement accuracy is. It is necessary to note the detection performance of the sonar. Its standard deviation of detection distance error is 1 5\% of the true distance and its standard deviation of detection angle error is 1 3 degrees. So we can analyze that if the target speed is $5 \mathrm{~m} / \mathrm{s}$, the distance between targets and sonar is $6000 \mathrm{~m}$, and the sampling period is $12 \mathrm{~s}$; then the standard deviation of the measurement distance is $90 \mathrm{~m}$ and the standard deviation of the angle error after converting is $104 \mathrm{~m}$. As is shown in the graph, Figure 1 shows ideal detection points, and Figure 2 shows artificial simulation points when the detection distance is $6000 \mathrm{~m}$.

At last, what is the meaning of large noise or great error? How can we describe them by mathematical methods? Then the formula can be expressed as

$$
\frac{\sigma \_ \text {measurement }}{V \cdot T} \geq 1
$$

where $\sigma$ measurement means the standard deviation of measurement, $V$ is the velocity of the target, and $T$ is the sampling period.

As mentioned before, if the sample time is $12 \mathrm{~s}$, then $90 /(5$ $\times 12)>1$ or $104 /(5 \times 12)>1$. It means that the measurement noise is large.

2.3. Clutter. In the underwater environment, certainly, there is a lot of clutter; so we have to consider the influence of the clutter. The presence of clutter deteriorates track initiation, reduces our initial accuracy, and even leads to failure of track starting.

\section{Traditional Algorithm Description}

3.1. The Heuristic Rule Method. Suppose that $\mathbf{r}_{i}, i=1,2, \ldots$, $N$, are position measurements from $N$ consecutive scans. The 


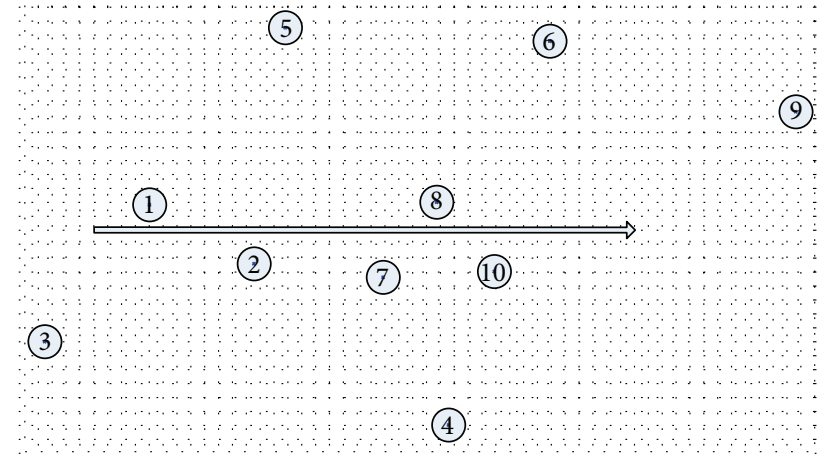

FIgURE 2: Artificial simulation distribution of sonar detection.

heuristic method initiates a track if any $M$ measurements from these $N$ scans satisfy the following requirements [12].

(1) The measured or estimated velocity is greater than a minimum value, $v_{\min }$, and smaller than a maximum value, $v_{\max }$.

(2) The measured or estimated acceleration is smaller than a maximum value, $a_{\max }$.

If there is more than one return, the one with the minimum acceleration is used to form the new track.

Mathematically, the two rules can be expressed as

$$
\begin{gathered}
v_{\min } \cdot T \leq\left|\mathbf{r}_{i}-\mathbf{r}_{i-1}\right| \leq v_{\max } \cdot T, \\
\left|\left(\mathbf{r}_{i+1}-\mathbf{r}_{i}\right)-\left(\mathbf{r}_{i}-\mathbf{r}_{i-1}\right)\right| \leq a_{\max } \cdot T^{2} .
\end{gathered}
$$

To reduce the possibility of forming false tracks, an optional, angle-limiting rule can be implemented in this heuristic track initiator as well. Let $\phi$ denote the angle between the vectors $\mathbf{r}_{i+1}-\mathbf{r}_{i}$ and $\mathbf{r}_{i}-\mathbf{r}_{i-1}$. Consider

$$
\alpha=\cos ^{-1}\left[\frac{\left(\mathbf{r}_{i+1}-\mathbf{r}_{i}\right) \cdot\left(\mathbf{r}_{i}-\mathbf{r}_{i-1}\right)}{\left|\left(\mathbf{r}_{i+1}-\mathbf{r}_{i}\right)\right|\left|\left(\mathbf{r}_{i}-\mathbf{r}_{i-1}\right)\right|}\right] .
$$

Then, the angle-limiting requirement can be expressed simply as

$$
|\alpha| \leq \phi
$$

where $\phi$ is the angle limitation for assumption of the straight motion target.

In the case that measurement data are ideal, the heuristic rule method is a good initiation method. But as shown in Figure 2, the measurement data look like a haphazard distribution. So after analyzing and demonstrating, we conclude that the heuristic rule method is not suitable to apply to the special underwater environment. Firstly, it is difficult to determine the appropriate value of $v_{\min }, v_{\max }$, and $a_{\max }$. Since the measurement data change a lot from time to time, we can set $v_{\min }$ as $0 \mathrm{~m} / \mathrm{s}$, but we hardly know the value of $v_{\max }$. If $v_{\max }$ is too big, then there is a lot of clutter included in the threshold, resulting in tracks initiation failure, and if it is too small, then the threshold cannot include the actual measurements. And it is more difficult for us to determine the right value of $a_{\max }$. At last, the angle-limiting is not suitable for the strong noise measurements.

So we conclude that the heuristic rule method cannot initialize a trajectory in the underwater environment with sonar sensor.

3.2. The Logic-Based (LB) Method. Let $z_{i}^{k}(t)$ be the $k$ th component of measurement $i$ at time $t$. The $k$ th component of the distance vector $\mathbf{d}_{i j}(t)$ between the measurements $\mathbf{z}_{i}(t)$ and $\mathbf{z}_{j}(t+1)$ is then defined as

$$
\begin{aligned}
d_{i j}^{k}(t)= & \max \left[0, z_{j}^{k}(t+1)-z_{i}^{k}(t)-v_{\max }^{k} t_{s}\right] \\
& +\max \left[0,-z_{j}^{k}(t+1)+z_{i}^{k}(t)+v_{\min }^{k} t_{s}\right],
\end{aligned}
$$

where $t_{s}$ is the time interval between the two scans. Assuming that the measurement errors are independent, normal, and zero-mean with covariance $R_{i}(t)$, the square normalised distance is then defined as

$$
D_{i j}(t)=\mathbf{d}_{i j}^{T}(t)\left[R_{i}(t)+R_{j}(t+1)\right]^{-1} \mathbf{d}_{i j}(t) .
$$

To associate the two measurements, $z_{i}(t)$ and $z_{j}(t+1), D_{i j}(t)$ is used as a test statistic which is compared to a predetermined threshold $\gamma$ based on a chi-squared distribution for a given degree of freedom. The searching procedure is carried out in the following fashion [12].

(i) Starting with a measurement (detection) from the first scan, an acceptance region which is always determined by (1) is set up for the second scan. For every measurement from the second scan falling into this region, a potential track is established.

(ii) For every potential track, consisting of two measurements, a straight-line extrapolation (first-order polynomial) is used to the third sampling time. The size of the acceptance region for measurements from the third scan is determined by using the prediction error covariance. The return from the third scan that is closest to the prediction is used to extend the potential track.

(iii) For every potential track, consisting of three or more returns, a second-order polynomial is used for prediction into the next scan. Again, the return closest to the prediction updates the potential track.

(iv) The procedure in (iii) is continued for a number of scans. The least squares method is used to estimate the residual (in order to determine the validating gate size). During the process, if no return is found in the acceptance region of a potential track, the track is terminated. Returns uncorrelated to any one of the tracks in each scan are used for starting new potential tracks as described in (i).

Under large measurement error or strong noise environment, it is not desirable to determine a trajectory only with two points and linearly predict the third point. Figure 2 appears to be haphazard distribution with less data. It is 
not easy to determine the covariance matrix in formula (6). $R_{i}(t)$ reflects the performance of the sensor, and we can get distance and angle information through sonar sensor. The raw sonar data are points with errors under polar coordinate; then we should transform those raw sonar data to points under rectangular coordinate. Because of this transformation process, the value of $R_{i}(t)$ changes, resulting in determining the true value difficultly. Even though there are a lot of papers that introduce how to solve the $R_{i}(t)$ in terms of approximation, we cannot get satisfactory approximate $R_{i}(t)$ in the underwater environment. After analyzing and simulating, we also conclude that the logic-based method cannot be applied directly to the underwater environment with large measurement errors. Our method is to modify the logicbased method in order to adapt it to the special underwater environment.

3.3. Hough Transform Method. There are a lot of advantages of Hough transform method to initialize trajectories. It has the stability and robustness for the slight measurement noise, and the accuracy is relatively high. But as mentioned earlier, in the underwater strong noise and heavy clutter environment, available data of sonar are relatively less with long delay. And we need to initialize a trajectory within two minutes. The simulation demonstrates that, by HT track initiation method, if the plan grid is set at a smaller number, it can form a track, but the path accuracy error is large, and if the number of the plan grid is set relatively large, it cannot form a trajectory. Because HT tracks initiation method is a batch method, so there are some disadvantages such as realtime performance, calculating capacity, and large memory requirement. The detection accuracy is influenced by discrete interval parameter constraints; HT method only determines if there is a line but cannot provide us with more information such as a much more complete description of the line, the relatively accurate endpoint position, and the length of the line.

\section{The Improved Sequential Method}

The proposed modified sequential initiation is introduced in this section. It is based on the logic-based method to deal with the specific underwater environment, and we estimate the initial states of targets with the least square method.

4.1. The Modified Logic-Based Method. In less than or equal to 10 successive sampling points, if there are 6 or more than 6 points which satisfy the threshold, it is considered that a track exists. This is the rule of the modified logic-based method. And if there are less than 6 in 10 points, then we are sure that no track exists.

Firstly, we will show several figures to illustrate the rules. In Figures 3 and 4, it shows the existence of a track. And in Figure 5, it shows nonexistence of a track.

Figures 3, 4, and 5 show track initiation process, and the corresponding formula of precise mathematical description is denoted as follows:

$$
\left|\mathbf{r}_{i+k}-\mathbf{r}_{i}\right| \leq v_{\max } \cdot k \cdot T+2 \sigma, \quad k=1,2,3,4,5,
$$

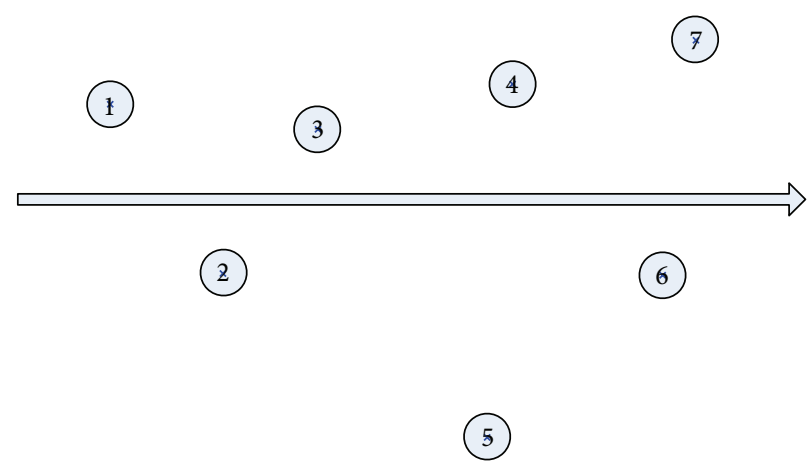

FIGURE 3: In the points sequence, 6 of 7 consecutive points satisfy the threshold (points 1, 2, 3, 4, 6, and 7 satisfy the threshold, and point 5 does not satisfy the threshold); then there is a track.

where $\mathbf{r}_{i+k}$ are the points of strong noise measurement at the $(i+k)$ th scan, $\mathbf{r}_{i}$ are the measurement data at the $i$ th scan, $k$ is the number of scans during the period from last scan to current scan that satisfies (7), $T$ is the time interval of the scan, and $\sigma$ is the 1 standard error of measurement distance of the sonar sensor.

Formula (7) describes the core of the modified logicbased method. In the underwater environment, we cannot ensure that we get the echo information of the target at every period and all the echo fall into the thresholds. So we use a variable $k$ to record the number of the scans during the period between two adjacent scans that meet the threshold requirement defined in (7). For example, $T$ is set to $12 \mathrm{~s}$ (in our simulation environment, the time interval of the scan is $12 \mathrm{~s}$ ) and the maximum speed is assumed to be $10 \mathrm{~m} / \mathrm{s}$. As shown in Figure 3, point 5 does not satisfy the threshold of point 4, but point 6 satisfies the threshold of point 4 ; then $k=2$, when $i=4$. And as shown in Figure 4, points 4 and 5 neither satisfy the threshold of point 3 , but point 6 satisfies the threshold of point 3 ; then $k=3$, when $i=3$. This record builds an adaptive threshold that can zoom in and out to adapt to strong noise measurement environment, especially when the maximum speed and the sampling period are relatively large.

As an example to further illustrate that, in the underwater environment, $T$ is set to $12 \mathrm{~s}$, and the maximum speed is assumed to be $10 \mathrm{~m} / \mathrm{s}$; then a target runs up to $120 \mathrm{~m}$ during the sampling period. One standard deviation sensor error is $1.5 \%$ of the actual distance. Then when the target distance is $6000 \mathrm{~m}, 1$ standard deviation of measurement is approximately $90 \mathrm{~m}$. Therefore, for the measurements with strong background noise, we need to consider two factors, kinematic factors of targets and sensors which we use, especially when the target distance is far; that is to say, $v_{\max } \cdot k \cdot T$ reflects the factors of kinematics, and $2 \sigma$ reflects the strong noise measurement factors. In general, in the underwater environment, multitarget tracks initiation is in far distance.

4.2. Least Squares Estimation of the Initial State. In multitarget tracking, after qualitatively determining the presence of a track, we also need to quantitatively determine the initial 
(4)

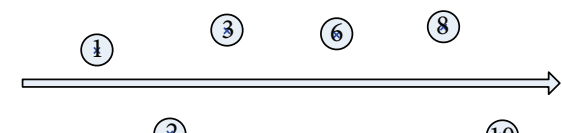

(2)

(10)

(7)

(5)

FigURE 4: In the points sequence, 6 of 10 consecutive points satisfy the threshold (points 1, 2, 3, 6, 8, and 10 satisfy the threshold, and points $4,5,7$, and 9 do not satisfy the threshold); then there is a track.

(2)

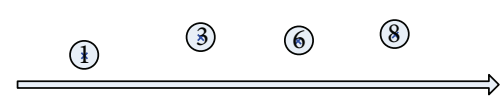

(10)

(

(5)

Figure 5: In the points sequence, only 5 of 10 consecutive points satisfy the threshold (points $1,3,6,8$, and 10 satisfy the threshold, and points 2, 4, 5, 7, and 9 do not satisfy the threshold); then there is no track.

state of this trajectory. In order to maintain high accuracy and stability during the trajectory tracking, there are many important factors to consider, including the initial state. If the initial state estimated is more accurate, subsequently the trajectory can be more quick to be in a stable state of high precision tracking, and if the estimation of initial state is inaccurate (position estimation error is large, or the speed estimation error is large), it will directly lead to losing the track. Therefore, initializing the state is also important for the multitarget tracking. For the traditional track initiation methods, such as the heuristic method, the logical method, or HT transform method, it is not necessary for us to give much attention to tracking initialization. If we can determine a track with the three methods, then the data are accurate enough to initialize a track with a simple and effective method. We can take the last point as the position of a target, accurately calculate the speed with the adjacent two points, and evaluate the acceleration with three points. Under the background of heavy noise, we need a new method which can deal with the large noise measurements. Three methods are considered to initialize a track: Kalman filter, linear fitting, and LSE. Theoretically, the Kalman filter estimation is the optimal method, but we only have a small number of data (six groups of data). It is hard to determine the initial state for the Kalman filter, and it cannot converge to the required accuracy with the limits of the small number of data. The linear fitting sometimes performs well, but it cannot work well with high probability of the heavy noise measurements.
After analyzing and simulating, we use the least squares method to estimate the initial state. We assume that $X$ is needed to be estimated, and its dimension is $n$. In general, we cannot get the real value of $X$ directly, but we can get the measurement information of linear combination of components of the vector $X$. Then $Z_{i}$ is the $i$ th measurement; we have

$$
Z_{i}=H_{i} X+V_{i}
$$

where $Z_{i}$ is the $m_{i}$ dimension vector, $H_{i}$ is the measurement matrix, and $V_{i}$ is noise matrix. If there are $r$ measurements, then

$$
\begin{aligned}
& Z_{1}=H_{1} X+V_{1} \\
& Z_{2}=H_{2} X+V_{2}
\end{aligned}
$$

$$
Z_{r}=H_{r} X+V_{r}
$$

Then we get the measurement formula:

$$
Z=H X+V \text {, }
$$

where $Z$ and $V$ are vector whose dimension is $m=\sum_{i=1}^{r} m_{i}$, and $H$ is $m \times n$ matrix.

If the rank of $H$ is $n$, then $H^{T} H$ is a positive definite matrix, and $m=\sum_{i=1}^{r} m_{i}>n$. So we estimate $X$ by the least squares method:

$$
\widehat{X}=\left(H^{T} H\right)^{-1} H^{T} Z \text {. }
$$

4.3. Program Flow Chart. The flow chart of the modified logic-based method is shown in Figure 6.

\section{Algorithm Description}

Step 1. Are there new sonar data? Yes, go to Step 2. No, stop this tracks initiation progress and wait for the next new sonar data.

Step 2. Is there a potential trajectory? Yes, go to Step 3; otherwise skip to Step 5.

Step 3. The sonar points match the potential trajectories; remove the successfully matching sonar points and update the potential trajectories; then skip Step 4 .

Step 4. Are there remaining sonar data? Yes, go to Step 5; otherwise skip to Step 6.

Step 5. Create new potential trajectories with remaining sonar data; go to Step 6.

Step 6. Delete the false potential trajectories.

Step 7. Are there sure trajectories? Yes, upgrade the potential trajectories to sure trajectories.

Step 8. The progress of tracks initiation finishes, and wait for the next new sonar data. 


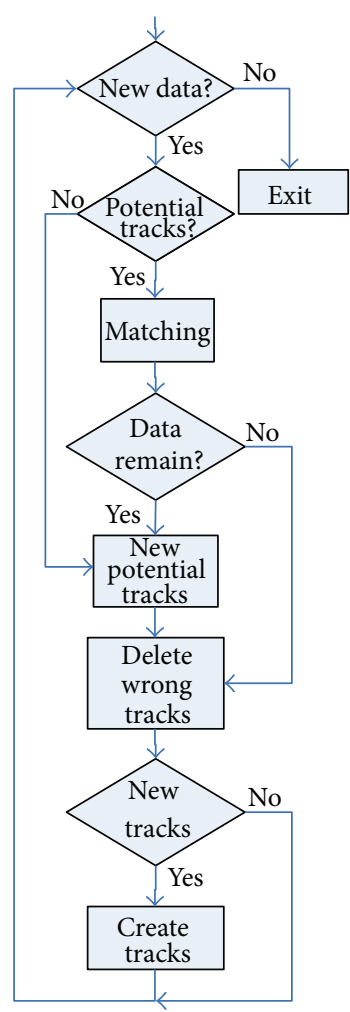

FIGURE 6: The flow chart of tracks initiation (the progress of upgrading potential trajectory to sure trajectory includes the tracks initialization).

\section{Simulation and Performance Analysis}

In this section, numerical simulations are conducted to verify the performance of the proposed algorithm. Consider a scenario of tracking multiple targets with an active sensor. Both the sensor and targets are assumed to be moving with constant velocities in the same plane, as shown in Figure 7.

Then, some important parameters are displayed below.

Sonar outputs: distance and angle of the targets.

Sampling period: $8 \mathrm{~s}$.

Space of simulation: a circular area of radius $10000 \mathrm{~m}$.

Distance measurement error: Gaussian distribution; 1 standard deviation is $1.5 \%$ of the actual measure distance.

Angle measurement error: Gaussian distribution; 1 standard deviation is 1 degree.

Real targets simulation: there are six targets, and the six targets make uniform linear motion, whose speed is $5 \mathrm{~m} / \mathrm{s}$; their initial position is $6000 \mathrm{~m}$ from the center to the distribution of a regular hexagon.

Detection platform: the speed is $4 \mathrm{~m} / \mathrm{s}$, and it moves run along the square, whose side is $400 \mathrm{~m}$.

Clutter: the number of clutter is 30 , and the position of the clutter obeys random distribution. The max distance of clutter is the maximum detection range.

As Figures 8, 9, 10, and 11 show, there is no delay, and we get the data from 400 simulations that each runs two minutes. Figure 8 shows the simulation statistical results.

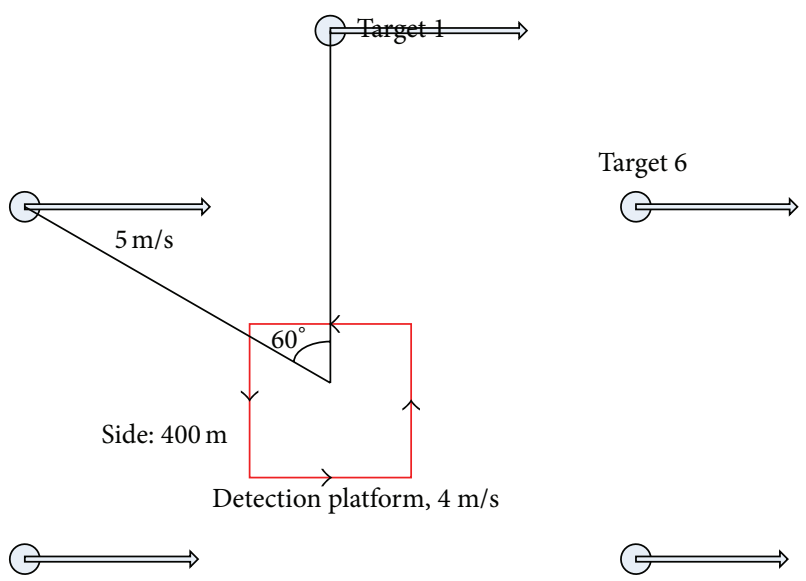

Target 5

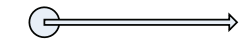

Target 4

FIgURE 7: Schematic diagram of platform and targets.

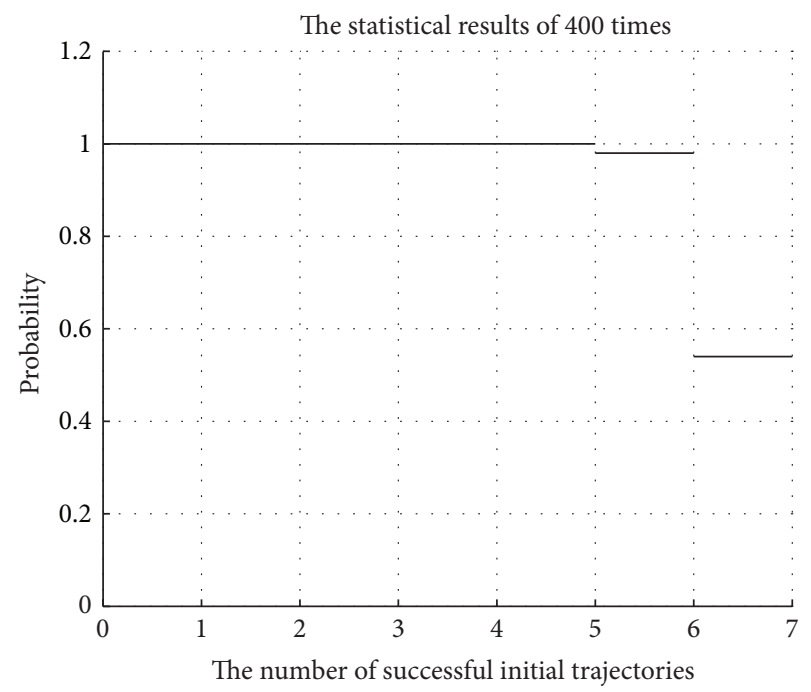

FIGURE 8: The probability of the number of successful initial trajectories.

From Figure 8, the modified logic-based tracks initiation method is efficient. Four or more than four of six trajectories were successfully initialized with the probability equal to 1. This is a very optimistic result for the underwater environment. And the probability that all the trajectories are initialized is 0.52 .

Consider the process of tracks initiation; not all trajectories simultaneously are formed. Figure 9 shows the time sequence at which trajectories are formed. The time is derived from the statistical simulation results. From Figure 9, 1.1 trajectories are formed at $72 \mathrm{~s}, 3.4$ trajectories are formed at $84 \mathrm{~s}, 5.3$ trajectories are formed at $96 \mathrm{~s}, 5.8$ trajectories are 


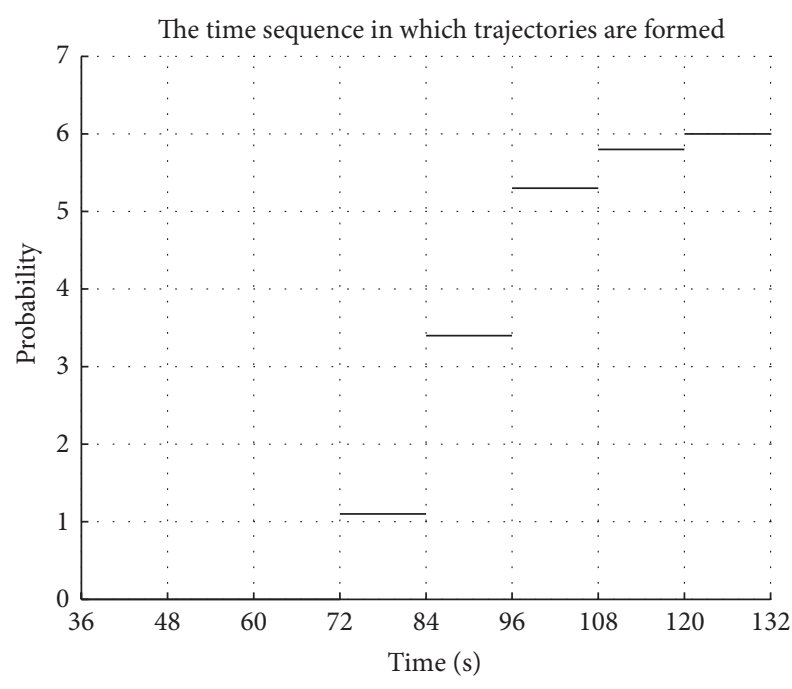

FIGURE 9: The time sequence in which trajectories are formed.

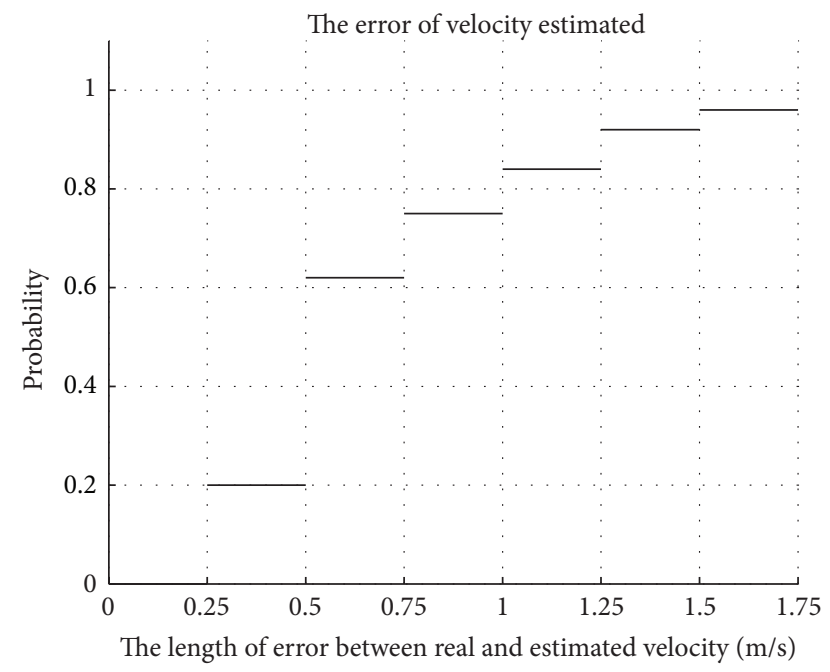

FIGURE 10: The length of error between real velocity and estimated velocity.

formed at $108 \mathrm{~s}$, and all the trajectories are formed at $120 \mathrm{~s}$. Then most of the trajectories are formed between $72 \mathrm{~s}$ and 96 s. More specifically, when the 7 th, 8 th, and 9th data are received, it is easier to form trajectories with high probability.

After determining the presence of a trajectory, we also need to estimate the initial state of the sure trajectory by the least squares method. There are six targets, and simulation time is two minutes. The number of simulations is 400 . Then we get the statistical result. The result is shown in Figures 10 and 11. From Figures 10 and 11, the error is small relatively, which satisfies the requirement of accuracy for subsequently stable tracking. For the same set of data, we initialized a track with the other three estimating methods and compared the errors, as shown in Table 1. From Table 1, we can conclude that the effect of LSE is better than linear fitting and KF.

From Figures 8, 9, 10, and 11 and Table 1, the modified logic-based method meets the requirements of multiple

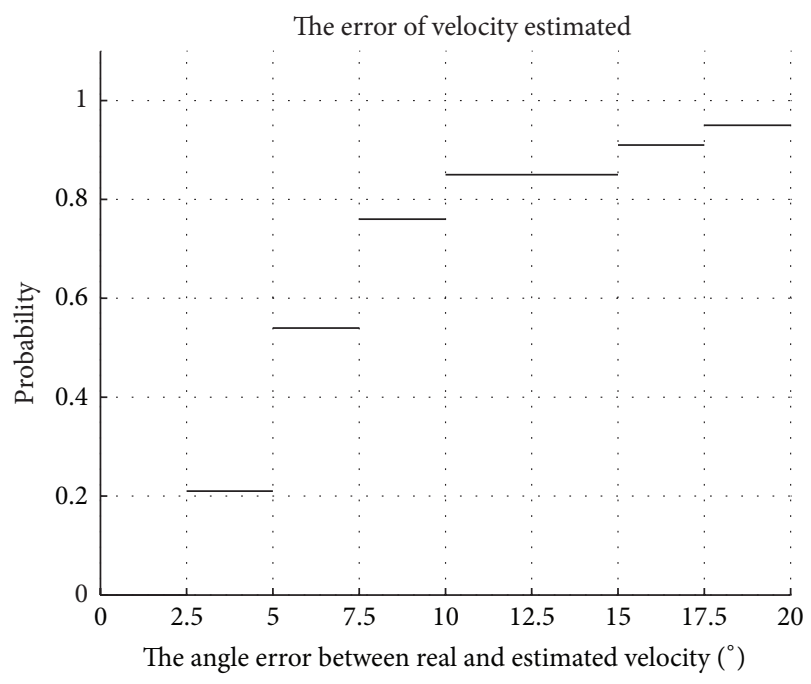

FIGURE 11: The angle error between real velocity and estimated velocity.

TABLE 1: The error analysis of the results of three initial methods.

\begin{tabular}{lccc}
\hline & Error of position & Error of speed & Error of angle \\
\hline LSE & $82.3 \mathrm{~m}$ & $0.46 \mathrm{~m} / \mathrm{s}$ & $8.6^{\circ}$ \\
Linear fitting & $114.5 \mathrm{~m}$ & $4.2 \mathrm{~m} / \mathrm{s}$ & $13.2^{\circ}$ \\
KF & $210.6 \mathrm{~m}$ & $8.5 \mathrm{~m} / \mathrm{s}$ & $12.7^{\circ}$ \\
\hline
\end{tabular}

targets tracking; that is to say, the estimated state meets the requirements of successively stable track.

\section{Discussion}

In this paper, we have proposed a novel tracks initiation algorithm to automatically initialize and maintain unknown number of tracks in the underwater environments, especially with the strong noise measurement. The new tracks initiation is that when at least 6 of 10 points meet the requirements, then we determine that there is a new track and the initial states of the parameters were estimated by the linear least square method. Compared to the conventional tracks initiation methods, our method not only considers the kinematics information of targets, but also regards the error of the sonar sensors as an important element. Computer simulations show that the proposed algorithm is valid in the complex environment of strong noise and clutter.

\section{Conflict of Interests}

The authors declare that there is no conflict of interests regarding the publication of this paper.

\section{Acknowledgment}

This work was supported by the Natural Science Foundation of China (no. 51309215). 


\section{References}

[1] Y. Bar-Shalom and T. E. Fortmann, Tracking and Data Association, Academic Press, San Diego, Calif, USA, 1988.

[2] I. J. Cox, "A review of statistical data association techniques for motion correspondence," International Journal of Computer Vision, vol. 10, no. 1, pp. 53-66, 1993.

[3] F. Dellaert, S. M. Seitz, C. E. Thorpe, and S. Thrun, "EM, MCMC, and chain flipping for structure from motion with unknown correspondence," Machine Learning, vol. 50, no. 1-2, pp. 45-71, 2003.

[4] G. Cybenko, V. H. Berk, V. Crespi, G. Jiang, and R. Gray, "An overview of process query systems," in Sensors, and Command, Control, Communications, and Intelligence (C3I) Technologies for Homeland Security and Homeland Defense III, 183, vol. 5403 of Proceedings of SPIE, pp. 183-197, Orlando, Fla, USA, April 2004.

[5] R. Luo, H. Min, and S. Lin, "Joint conditional random fields for multi-object tracking with a mobile robot," Robot, vol. 33, no. 3, pp. 279-286, 2011 (Chinese).

[6] Y. Bar-Shalom and T. E. Fortmann, Tracking and Data Association, Academic Press, 1988.

[7] Y. Bar-Shalom, K.-C. Chang, and H. M. Shertukde, "Performance evaluation of a cascaded logic for track formation in clutter," IEEE Transactions on Aerospace and Electronic Systems, vol. 25, no. 6, pp. 873-878, 1989.

[8] X. Chen, J. Lin, and J. Zhang, "Performance analysis of track initiation algorithm," in Proceedings of the 6th World Congress on Intelligent Control and Automation (WCICA '06), pp. 42394243, Dalian, China, June 2006.

[9] B. D. Carlson, E. D. Evans, and S. L. Wilson, "Search radar detection and track with the Hough transform," IEEE Transactions on Aerospace and Electronic Systems, vol. 30, no. 1, pp. 102-108, 1994.

[10] M. C. Smith and E. M. Winter, "Feature space transform for multi target detection," in Proceedings of the IEEE Conference On Decision and Control, pp. 835-836, Albuquerque, New Mexico, 1980.

[11] T. Lo, H. Leung, A. W. Bridgewater, and J. Litva, "Multitarget track initiation using a modified Hough transform," in AGARD Conference Proceedings, vol. 539 of Pointing and Tracking Systems, pp. 25.1-25.8, 1994.

[12] H. Leung, Z. Hu, and M. Blanchette, "Evaluation of multiple target track initiation techniques in real radar tracking environments," IEE Proceedings-Radar, Sonar and Navigation, vol. 143, no. 4, 1996. 


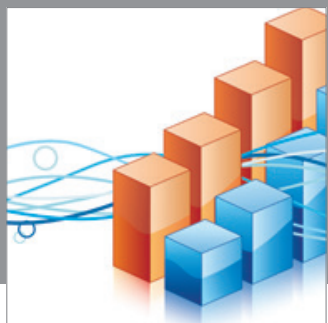

Advances in

Operations Research

mansans

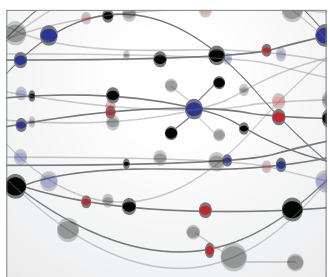

The Scientific World Journal
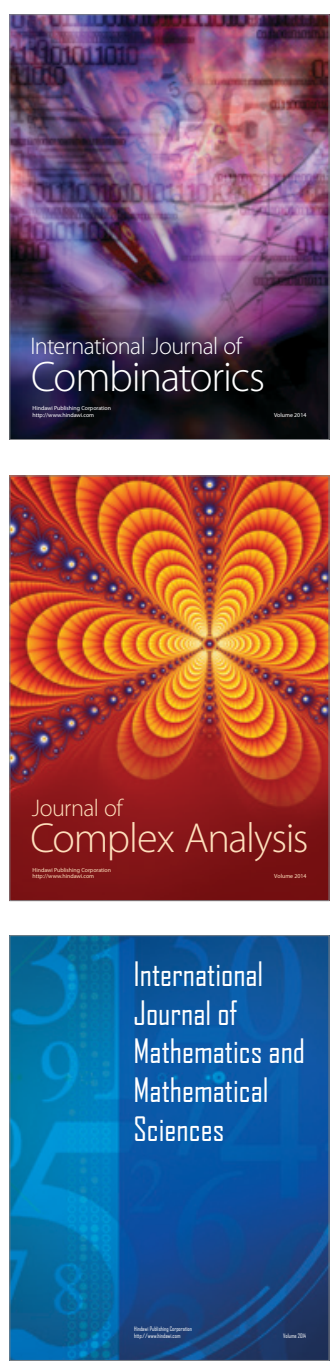
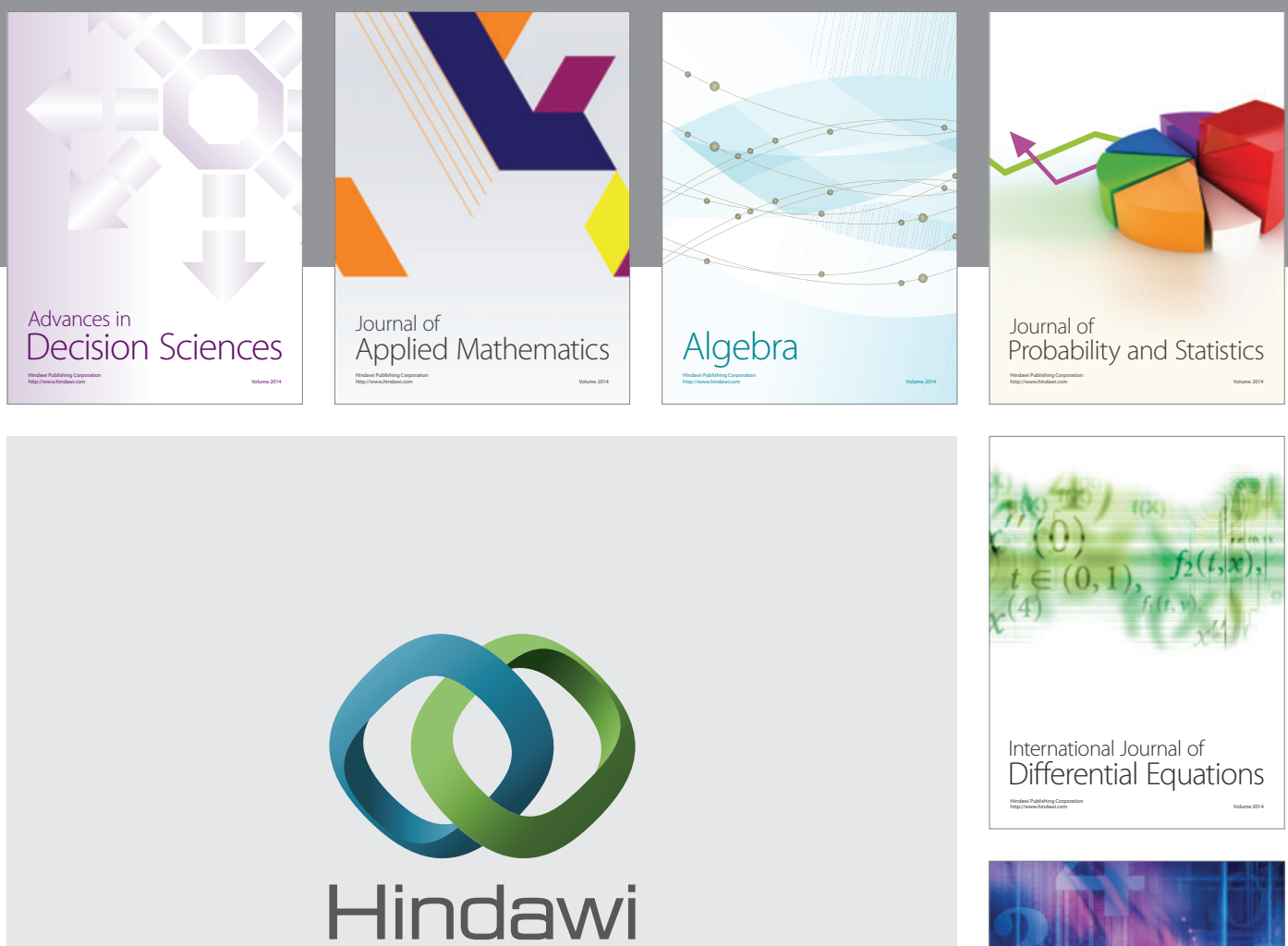

Submit your manuscripts at http://www.hindawi.com
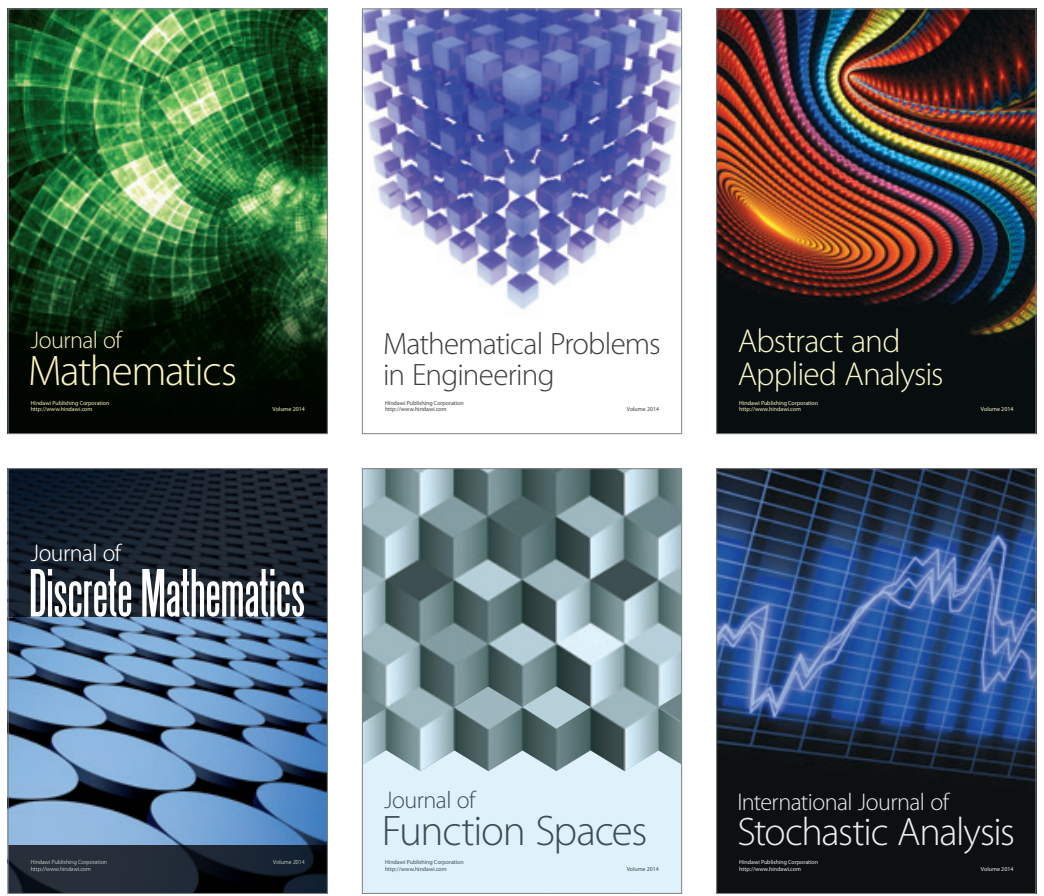

Journal of

Function Spaces

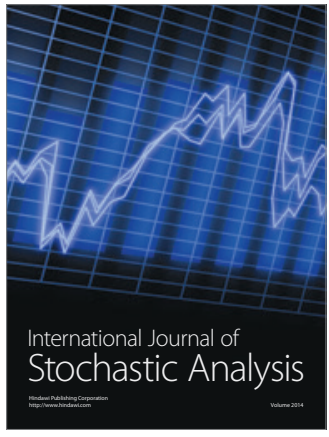

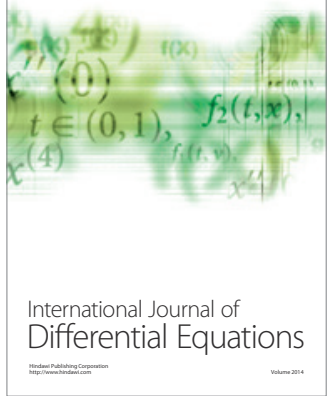
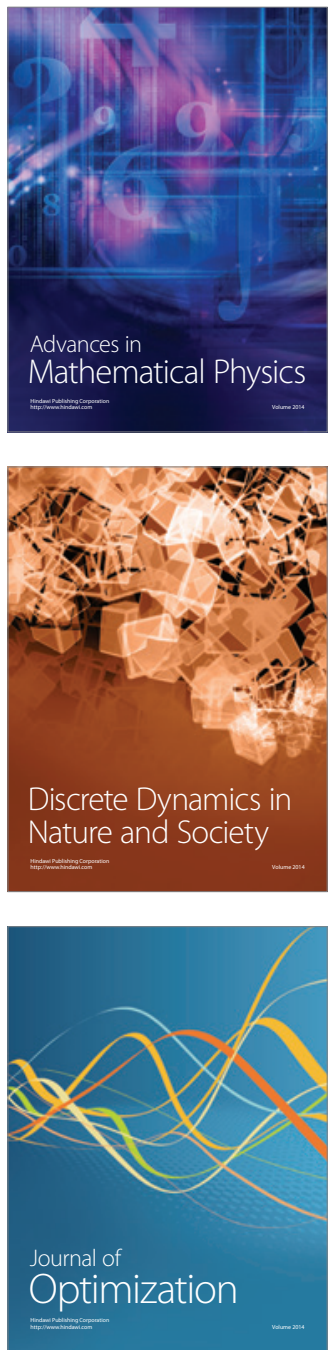